\title{
Pattern of Parent Participation in Motor Training for Children with Cerebral Palsy: A Qualitative Research Tianping Zheng ${ }^{1, a}$ and Tao Wang ${ }^{1, b,{ }^{*}}$ \\ ${ }^{1}$ Special Education Department, Chongqing Normal University, Chongqing, China \\ a1163915828@qq.com, bwangtao.cq@163.com \\ ${ }^{*}$ Corresponding author
}

\begin{abstract}
By using the method of qualitative research through observation parents participating in rehabilitation training for children with cerebral palsy in educational system, complemented with the parent interviews, this paper discussed the pattern of parent participation in motor training for children with cerebral palsy and the influence of the parent participation pattern on training effect under the background of education. The pattern of parent participation in motor training was divided into the assisted participation, the executive participation, and the interactive participation. In the three patterns, the improvement of motor ability of children with cerebral palsy was the most significant under the interactive participation pattern.
\end{abstract}

Key words: Children with cerebral palsy, Parent participation, Participation pattern, Qualitative research.

\author{
脑瘫儿童家长参与动作训练模式的质性研究$$
\text { 郑天平 }{ }^{1, a} \text {, 王滔 }{ }^{1, \mathrm{~b},{ }^{*}}
$$ \\ 1 重庆师范大学特殊教育系, 重庆, 中国 \\ a1163915828@qq.com, bwangtao.cq@163.com \\ *通讯作者
}

中文摘要. 运用质性研究的方法, 通过实地 观察家长参与脑瘫儿童康复训练的情况并 结合对家长的深度访谈, 探讨在教育背景下 家长参与脑㿈儿童动作训练的模式, 以及家 长参与模式对脑瘫儿童训练效果的影响。根 据家长参与动作训练的角色不同, 可以将其 参与模式分为家长辅助式参与、家长执行式 参与、家长互动式参与。在三种模式中, 家 长互动式参与模式下的脑㿈儿童动作能力 的改善最为显著。
关键词: 脑㿈儿童; 家长参与; 参与模式; 质性研究

\section{1. 引言}

脑瘫是指中枢神经系统受损而引起的 非进行性疾病, 主要表现为运动功能障碍及 姿势异常, 同时可能并发多种合并症 ${ }^{[1]}$ 。我 国0-6岁儿童的脑瘫发病率为1.86/1000, 目 前有0-6岁脑痽患儿31万多 ${ }^{[2]}$ 。国内外尚无 
一种治愈脑痽的特效药物, 认为在众多的方 法中, 动作训练最为有效 ${ }^{[3]}$ 。脑㿈儿童的动 作训练是一个需要长期坚持的过程, 仅靠专 业康复师短暂的动作训练是远远不够的。日 常生活占了脑瘫儿童生活的大部分, 因此需 要家长在日常生活中创造有利于脑㿈儿童 学习和克服心理障碍的环境, 并通过康复老 师给予的培训和指导, 长期对脑㿈儿童进行 康复训练 ${ }^{[4]}$ 。

家长参与主要是指以家庭或者机构为 训练场所, 家长在专业人员提供理论知识和 教育培训后, 参与到脑㿈儿童动作训练的过 程中, 包括评估、计划拟定、动作训练, 日 常护理, 以及给予其心理和社会关怀, 以提 高和巩固脑㿈儿童动作训练效果 ${ }^{[4-7]}$ 。国内 外不少研究发现, 医疗系统中渗透家长参与 脑瘫儿童康复训练能明显提高脑瘫儿童的 治疗效果, 且在出院后家长能长期坚持做康 复训练的儿童病情更加稳定 ${ }^{[8,9]}$ 。家长参与 脑瘫患儿康复训练的时间、投入的精力, 都 会影响到儿童的康复效果 ${ }^{[1,10]}$ 。家长作为陪 伴脑瘫儿童时间最长的人, 为其营造温暖安 全的成长环境, 参与其康复训练的过程是很 重要的 ${ }^{[11]}$ 。家长还可以通过各种游戏、娱 乐活动缓解脑瘫儿童的不良情绪, 给予他们 更多的心理和社会关怀 ${ }^{[12]}$ 。虽然已有研究 用可靠的数据证实了家长参与的作用, 但是 对家长参与的模式和特点思考还比较少。基 于此, 我们采用质性研究的方法, 探讨在教 育背景下家长参与脑瘫儿童康复训练的模 式、特点及其效果。

\section{2. 研究方法}

\section{1 研究对象}

实地观察对象: 本研究选取了2014年11 月 2015年1月一直在我校动作训练中心做 动作训练的五位脑瘫儿童的家长。其中三位 是脑㿈儿童的母亲, 平均年龄35岁, 中学文 化程度, 全职照料孩子; 另外两位分别是脑 瘫儿童的外婆和婆婆, 平均年龄58岁, 小学 文化程度。

深度访谈对象: 包括上述五位实地观察 的对象, 以及两位定期带脑㿈儿童来中心评 估的家长, 两位均是母亲。

\section{2 研究方法}

实地观察: 每天在动作训练中心的大教 室里实施观察, 主要观察家长在动作训练过 程中的参与情况, 包括家长的动作操作, 家 长的态度, 家长的情绪和行为表现, 与自己 孩子的沟通, 以及家长、脑痽儿童与康复老 师之间的互动等。为了方便观察到每个对 象, 选取视野较好的地方, 如面对镜子或者 面朝大家的地方进行观察, 在现场记录下主 要事件并写下关键词。

深度访谈: 为了弥补实地观察的局限, 了解家长对于参与动作训练的内心想法和 观念, 在中心的家长接待室对家长进行了深 度访谈, 每人每次访谈的时间大约 30 分钟左 右。具体的访谈提纲主要涉及四个方面：一 是家长的基本情况, 二是家长对家长参与的 观念和态度, 三是家长对参与动作训练的自 我效能, 四是回到家里家长对于孩子的日常 养护情况。

\section{3 资料的整理和分析}

资料的整理: 观察资料是每晚根据现场 记录的事件和关键词进行情景再现后补充 记录, 并把一些当时的想法以及场景的解读 写在记录的后面。对于观察中出现的一些疑 问也做出记录, 以方便在以后的观察和访谈 中找到答案。访谈资料则是在访谈结束后, 将手机中的录音逐字转录成文字资料。

资料的分析: 在实地观察和深度访谈结 束后，采用Strauss的方法将所得的文字资料 编码、整理和归类, 具体做法是: 搜集初步 资料, 写成札记, 一行行读札记 ${ }^{[13]}$, 在段 落中找出或总结出关键词, 并进行编码。

\section{3. 研究结果与分析}

\section{1 家长参与的模式}

\section{1 .1 辅助式参与模式}

这类家长期望脑㿈儿童得到进步, 主动 接送脑瘫儿童上康复训练课, 并把日常生活 中遇见的问题反映给康复老师。家长基本上 把希望寄托在康复老师上, 这种依赖心理导 致她们没有把家长参与康复训练渗透到日 常生活中, 其参与行为大多数表现为把孩子 
送到课堂, 协助、陪护照看孩子的日常生活 事宜, 如在康复老师上课时给脑㿈儿童喂 水, 帮助上则所等。

辅助式参与模式的特点主要有: (1)对家 长参与的认识不够, 态度上不积极, 参与的 主动性较低, 只是在老师有要求时做一些辅 助性工作。(2)家长部分参与到脑㿈儿童动作 训练中, 参与行为较少, 是低程度的家长参 与。(3)参与效果上难以发挥自己对孩子康复 训练的作用, 在脑㿈儿童康复训练中的自我 效能感不高。其模式见图1。

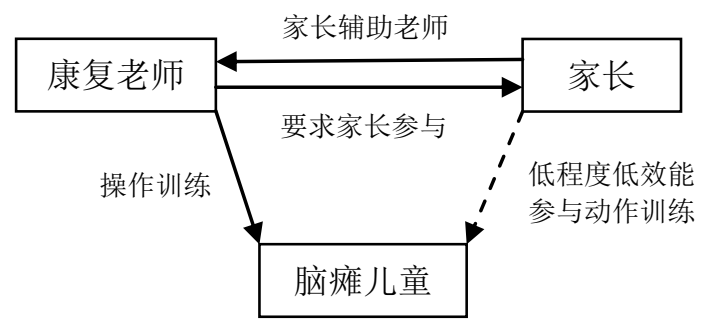

图1 家长辅助式参与模式图

\subsection{2 执行式参与模式}

在这类家长参与模式中, 康复老师定期 评估和拟定动作训练计划, 由家长自己独立 执行操作训练, 达到短期目标后再次由康复 老师进行评估, 调整训练计划。在每次评估 和确定训练计划之后, 康复老师会用两天的 时间, 对家长实施高强度的本次训练计划的 针对性训练, 确保家长能够独立执行老师制 定的训练计划。这种模式中的家长通常接受 过长期的专业指导, 累计了较多的实践经 验, 掌握了一定的操作技巧。

执行式参与模式的特点主要有: (1)对家 长参与有较为明确但不全面的观念和认识, 态度积极, 由家长独立操作训练计划, 参与 的主动性较强。(2)家长的参与行为较多, 不 仅要执行康复老师制定的动作训练计划, 而 且在日常生活中也会注意到与脑㿈儿童康 复有关的问题, 定期带孩子做评估, 并向康 复老师反馈自己遇到的问题, 是高程度的家 长参与。(3)如果孩子有进步或者说没有退 步, 家长参与的自我效能感就较高, 但是由 于脑痽儿童的康复训练计划是由家长独立 完成, 对于训练的执行还缺乏灵活性, 而康 复老师不能够随时根据孩子的变化做出调 整, 一些问题无法及时得到解决, 以致衍生
出某些代偿性问题, 会显著降低家长参与的 自我效能感。其模式见图2。

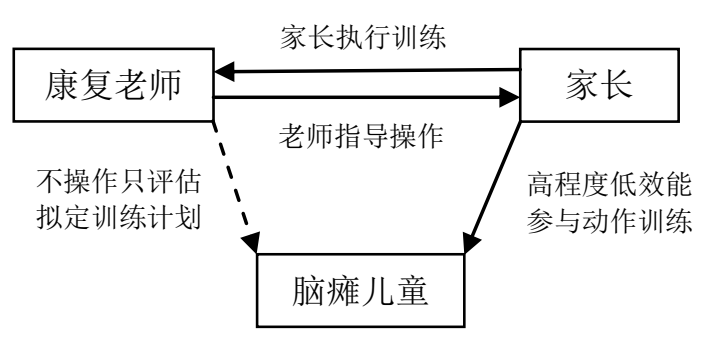

图2 家长执行式参与模式图

\subsection{3 互动式参与模式}

在这类家长参与模式中, 康复老师评 估、制定训练方案，康复老师和家长在时间 上互补地实施康复训练计划, 最后再由康复 老师评定实施的效果, 对训练方案做出调 整。在该过程中康复老师针对脑瘫儿童的问 题以及家长的操作手法给予实时的指导, 家 长则主动参与到康复老师的训练课程, 并将 家长参与渗透到日常生活, 就脑㿈儿童的问 题主动沟通, 征询老师意见。

互动式参与模式的特点主要有: (1)家长 参与的主动性高, 态度积极, 对家长参与有 明确清晰的观念, 主动参与训练过程, 并主 动找老师解决问题。(2)参与行为较多, 从评 估、制定方案, 到操作训练、反馈都有家长 的参与, 康复老师在操作过程中与家长及时 沟通、互相协作, 实时调整训练计划与训练 重点, 是高程度的家长参与。(3)家长参与动 作训练的全程, 家长和老师共同为孩子的进 步负责, 共同见证脑痽儿童的进步, 家长的 自我效能感明显高于以上两种模式。其模式 见图3。

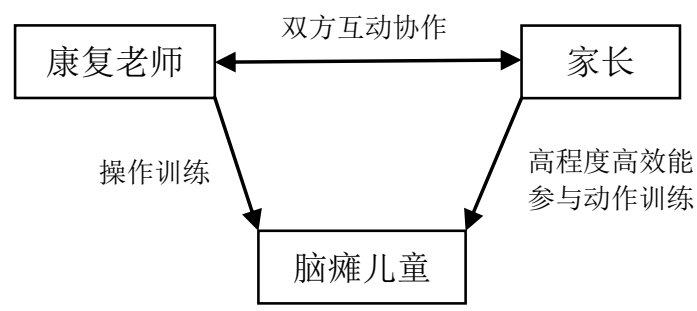

图3 家长互动式参与模式图

\section{2 家长参与脑瘫儿童康复训练的效果}

采用全人疗育评估记录表中的表二, 比 较三种家长参与模式下的七位脑痽儿童在 3 个月观察期前后的现有能力的变化, 以考 
察家长参与模式对脑瘫儿童动作能力改善 的影响。脑瘫儿童观察前后的现有能力、短 期和长期目标见表 1 。

表1 观察前后脑痽儿童的现有能力及目标

\begin{tabular}{|c|c|c|c|c|c|}
\hline \multirow{2}{*}{$\begin{array}{l}\text { 家长参 } \\
\text { 与模式 }\end{array}$} & \multirow{2}{*}{ 姓名 } & \multicolumn{3}{|c|}{3 个月前 } & \multirow{2}{*}{$\frac{\text { 3个月后 }}{\text { 现有能力 }}$} \\
\hline & & 现有能力 & 短期目标 & 长期目标 & \\
\hline \multirow{3}{*}{$\begin{array}{l}\text { 互动式 } \\
\text { 家长参 } \\
\text { 与模式 }\end{array}$} & TML & $\begin{array}{l}\text { 双手扶物 } \\
\text { 跍走 } 3 \text { 步 }\end{array}$ & $\begin{array}{l}\text { 单手扶物 } \\
\text { 站立 } 5 \text { 秒 }\end{array}$ & $\begin{array}{l}\text { 单手扶物 } \\
\text { 跪走3步 }\end{array}$ & $\begin{array}{c}\text { 左手扶物站 } \\
\text { 立交替抬脚 } \\
3 \text { 下 }\end{array}$ \\
\hline & TMZ & $\begin{array}{c}\text { 独立高跪2 } \\
\text { 秒 }\end{array}$ & 独立站 2 秒 & 跨立 & $\begin{array}{c}\text { 左手扶物站 } \\
\text { 立交替抬脚 } \\
3 \text { 下 }\end{array}$ \\
\hline & HYQ & $\begin{array}{l}\text { 独立交替 } \\
\text { 半踭3下 }\end{array}$ & $\begin{array}{l}\text { 独立行走 } \\
\text { 左右各1步 }\end{array}$ & $\begin{array}{c}\text { 独立行走3 } \\
\text { 步 }\end{array}$ & $\begin{array}{c}\text { 独立行走3 } \\
\text { 步 }\end{array}$ \\
\hline \multirow{2}{*}{$\begin{array}{l}\text { 辅助式 } \\
\text { 家长参 } \\
\text { 与模式 }\end{array}$} & $\mathrm{LZ}$ & 蹲走3步 & $\begin{array}{c}\begin{array}{c}\text { 左单脚站3 } \\
\text { 秒 }\end{array} \\
\end{array}$ & 蹲跳 & $\begin{array}{l}\text { 左单脚站 } 3 \\
\text { 秒 }\end{array}$ \\
\hline & HL & $\begin{array}{c}\text { 独立高跪5 } \\
\text { 秒 }\end{array}$ & 独立站5秒 & 跨步站2秒 & $\begin{array}{c}\text { 独立高踠20 } \\
\text { 秒 }\end{array}$ \\
\hline \multirow{2}{*}{$\begin{array}{l}\text { 执行式 } \\
\text { 家长参 } \\
\text { 与模式 }\end{array}$} & LR & 蹲走 & $\begin{array}{c}\text { 左单脚站5 } \\
\text { 秒 }\end{array}$ & $\begin{array}{c}\text { 右单脚站5 } \\
\text { 秒 }\end{array}$ & 蹲走 \\
\hline & WSW & 独立高跍 & $\begin{array}{c}\text { 单手扶物 } \\
\text { 站立 }\end{array}$ & 独立趾走 & 独立高䟦 \\
\hline
\end{tabular}

在全人疗育评估记录表中, 每一个短期 和长期目标都是治疗师根据个案现有能力 而设定的, 需要脑瘫儿童在 1 个月和 3 个月中 达到。从表1可见, 在互动式家长参与模式 下的3位儿童 3 个月之后的现有能力都有所 改善。尽管TML单手扶物站立交替抬脚 3 下 的能力不完整, 但还是在进步, 达到了观察 前的短期目标; TMZ的长短期目标虽然并 未达成, 但现有能力也在朝着评估表的方向 上有所进步; 而 HYQ的能力从骨盆阶段开 始跨入到下肢阶段, 长短期目标都达到了。

在辅助式家长参与模式下的两位儿童3 个月之后的能力同样也都有所改变, 但改善 不够。LZ达到了短期目标, HL的长短期目 标没有达到, 但其高䟜姿的维持时间比之前 长了15秒。而在执行式家长参与模式下, 两 位脑瘫儿童观察前后的现有能力并未发生 改变, WSW甚至在3个月之后还出现了骨盆 处的异常肌肉张力。综合比较个案观察前后 的能力变化情况, 互动式家长参与模式下的 脑瘫儿童的进步最为明显。

\section{4. 讨论}

\section{1 三种家长参与模式的不同作用}

在脑瘫儿童的动作训练中, 康复老师每 天的操作时间是儿童日常生活时间的小部 分, 而且老师的训练剂量也是有限的, 这就 使家长参与在脑痽儿童康复中起着重要的 作用, 不同的家长参与模式具有不同的特点 和效果。辅助式家长参与模式下的脑㿈儿童 能够长期得到康复老师专业的服务, 但家长 在课上和课后较低的参与度, 浪费了很多康 复训练时间。这种模式中家长低程度低效能 的参与, 导致脑瘫儿童康复训练的进程相对 较慢, 所花时间较多。

执行式家长参与模式下的脑瘫儿童能 够在自然放松的环境中进行康复训练, 家长 可以自由安排操作的时间及时长。虽然家长 每天都花很多时间给脑瘫儿童做训练, 但这 种高程度低效能的参与离开了康复老师的 实时指导, 家长不能够灵活地调节康复训练 方案, 会衍生出一些其他代偿问题, 使得脑 瘫儿童难以得到明显的进步。

在互动式家长参与模式下, 康复老师与 家长在训练时间上互补, 对于存在的问题相 互沟通, 紧密协作, 并且家长在与老师的互 动中得到不断学习, 这种高程度高效能的参 与模式可以让脑瘫儿童的进步事半功倍。通 常, 辅助式或执行式模式中的家长经过长期 的摸索, 当他们的观念清晰、具有一定的操 作手法、看到孩子的进步、并对康复老师充 满信任的时候, 会逐渐转变成互动式的参与 模式。

\section{2 家长参与脑㿈儿童动作训练的局限}

脑瘫儿童家长参与动作训练的最大问 题就是家长的心态及其与孩子的互动沟通。 脑痽康复不是一朝一夕的事情, 家长日复一 日地帮助孩子做训练, 难免也会觉得枯燥乏 味, 而孩子进步缓慢, 加之孩子调皮、不专 心, 常常使得家长对孩子的态度不好, 最后 发展到大吼大叫、威胁甚至打骂。

脑瘫儿童已有的进步以及家长的期望 会促使家长参与康复训练, 但他们更关注脑 瘫儿童动作上的问题。大多数家长会把康复 老师的要求 “贯彻始终” , 甚至是高标准严 要求, 几乎把所有的时间都花在了孩子的康 
复训练上, 很少有家长会考虑到脑瘫儿童的 体能、以及情绪心理和社会关怀的需求。家 长倾向于把出现的一切问题归因为脑瘫儿 童的不努力、不上进、不专心, 导致家长对 脑瘫儿童的态度消极, 缺乏理解, 很难与孩 子互动和沟通。

\section{3 本研究的局限及未来研究方向}

本研究主要采用了实地观察和深度访 谈的质性研究方法, 这两种方法获得的资料 容易受到研究者主观因素的影响, 并且实地 观察时只记下了关键字词, 通过再现情景完 善资料, 可能会有一些细节被遗忘, 导致资 料不够完整和客观。

\section{5. 结束语}

在观察和访谈中我们发现家长的训练 经验、教育水平、教养方式、情绪状态等都 会影响到家长对动作训练的参与模式, 今后 将进一步研究脑痽儿童家长参与动作训练 模式的影响因素及其关系。

\section{致谢}

本文为国家社科基金西部项目《残疾儿 童心理健康及其与亲职压力关系的研究》 (13XRK002)的阶段性成果之一。

\section{References}

[1] H. Yan, The importance of family involvement in rehabilitation of cerebral palsy, Chinese Journal of Clinical Rehabiliation, vol. 6, p. 2910, 2002.

[2] N. X. Huang, The professional doctors guide parents to participate in the rehabilitation training for cerebral palsy in children, Practical Journal of Medicine \& Pharmacy, vol. 29, pp. 324-325, 2012.

[3] H. M. Mao, J. Zhang, and W. Hua, Effect of patients' participation on evaluating the rehabilitation treatment of the children with cerebral palsy, Clinical Nursing Journal, vol. 7 , pp. 7-8, 2008.

[4] P. Vasli, M. Salsali, Parents' participation in taking care of hospitalized children: A concept analysis with hybrid model, Iranian Journal of
Nursing and Midwifery Research, vol. 19, pp. 139-144, 2014.

[5] X. L. Zhou, J. B. Shen, and L. Feng, et al., Analysis of the effect of comprehensive rehabilitation treatment for children with spastic paralysis by parent involvement, Modern Rehabilitation, vol. 5, p. 84, 2001.

[6] Y. Zhang, D. Qu, and Y. J. Zhang, et al., Case study on the rehabilitation training in participation of parents for a child with cerebral palsy, Journal of Practical Medical Techniques, vol. 13, pp. 4026- 4072, 2006.

[7] S. Liang, and C. X. Gao, Study on patriarch joined cooperative rehabilitation mode in cerebral palsy, Modern Rehabilitation, vol. 5, pp. 76-77, 2001.

[8] C. X. Gao, S. M. Zhang, and X. L. Wang, The observation of the effect of parent involvement in the rehabilitation of cerebral palsy with cerebral palsy in 64 cases, Medical Theory and Practice, vol. 16, pp. 1179-1180, 2003.

[9] J. A. Skelton, M. B. Irby, and B. M. Beech, et al., Perceptions of attrition and family participation: A qualitative study of pediatric obesity clinicians, Academic pediatrics, vol. 12, pp. 420-428, 2012.

[10] X. H. Yao, Clinical trial of parents' role in rehabilitation of children with cerebral palsy, Chinese Pediatrics Integrated Traditional and Western Medicine, vol. 1, p. 30, 2010.

[11] J. L. Chang, Y. J. Xu, and B. Sun, Effect of parents participation in evaluating the rehabilitation treatment of infantile cerebral palsy, Chinese Journal of Clinical Rehabilitation, vol. 9, pp. 158- 159, 2005.

[12] D. M. Zhang, and C. D. Zhang, The effect of parent involvement training on the rehabilitation effect of cerebral palsy in children, Medical Journal of Chinese People Health, vol. 21, pp. 1394-1428, 2009.

[13] M. B. Miles, and A. M. Huberman, Qualitative data analysis : Method and practice, Chongqing: Chongqing University Press, pp. 83-86, 2008. 